\title{
Organización del almacén de garantías de una empresa distribuidora automotriz de Ciudad Obregón
}

\section{Organization of the warehouse of guarantees of an automotive distribution company of Obregon City}

\author{
BELTRÁN-ESPARZA, Luz Elena†*, GONZÁLEZ-VALENZUELA, Elizabeth, FORNÉS-RIVERA, \\ René Daniel y GODOY-BOJÓRQUEZ, Fabiola Berenice
}

Instituto Tecnológico de Sonora

ID $1{ }^{\mathrm{er}}$ Autor: Luz Elena, Beltrán-Esparza / ORC ID: 0000-0003-2755-812X, Researcher ID Thomson: G-5037-2018, arXiv Author ID: luzelena_beltran, CVU CONACYT ID: 276136

ID $1^{\text {er }}$ Coautor: Elizabeth, González-Valenzuela / ORC ID: 0000-0003-3774-5324, Researcher ID Thomson: G-5042-2018, arXiv Author ID: elizabeth_gonzalez_v, CVU CONACYT ID: 276316

ID $2^{\text {do }}$ Coautor: René Daniel, Fornés-Rivera / ORC ID: 0000-0002-7438-0056, Researcher ID Thomson: G-3906-2018, arXiv Author ID: rene_fornes, CVU CONACYT ID: 280435

ID $3^{\text {er }}$ Coautor: Fabiola Berenice, Godoy-Bojórquez / ORC ID: 0000-0003-3700-2598, arXiv Author ID: fabiola_godoy

\section{Resumen}

Este proyecto se realizó en el almacén de garantías de una empresa distribuidora automotriz en Ciudad Obregón, el cual presentaba la desorganización de piezas, archivos innecesarios, pisos obstruidos y falta de limpieza, provocando que se extendieran el tiempo de búsqueda de piezas y archivos. Por lo tanto, se estableció el objetivo de mejorar las condiciones de organización y limpieza del almacén a través de la filosofía 5’S. El procedimiento consistió en: describir el área bajo estudio; evaluar la situación inicial del almacén; capacitar al personal; elaboración de inventario de objetos del almacén; separación de objetos necesarios e innecesarios; posteriormente se asignó un lugar a cada objeto, en seguida se limpió el área y objetos del almacén ; se elaboraron estándares de las actividades que se realizan en el almacén; se midió el nivel de cumplimiento de la filosofía 5?S y finalmente se evaluó la situación final del almacén. Inicialmente, la situación del almacén en cuanto criterios de 5 ?S tuvo un $16.47 \%$ de cumplimiento y una vez implementada la filosofía $5 ? \mathrm{~S}$, se incrementó la calificación a $92.94 \%$, por lo tanto, se logró el objetivo de este proyecto que era mejorar las condiciones de organización y limpieza del almacén.

\begin{abstract}
This project was carried out in the warehouse of guarantees of an automotive distribution company in Obregon City, which presented the disorganization of parts, unnecessary files, clogged floors and lack of cleanliness, causing the time to search for parts and files to be extended. Therefore, the objective of improving the conditions of organization and cleanliness of the warehouse was established through the 5'S philosophy. The procedure consisted of: describing the area under study; evaluate the initial situation of the warehouse; train the staff; elaboration of inventory of warehouse objects; separation of necessary and unnecessary objects; subsequently a place was assigned to each object, then the area and objects of the store were cleaned; standards were developed for the activities carried out in the warehouse; the level of compliance with the 5'S philosophy was measured and finally the final warehouse situation was evaluated. Initially, the situation of the warehouse as criteria of 5'S had a $16.47 \%$ compliance and once implemented the philosophy 5'S, the rating was increased to $92.94 \%$, therefore, the objective of this project was achieved that was to improve the conditions of organization and cleaning of the warehouse.
\end{abstract}

Mejora, Organización, $5 \mathrm{~s}$

Improvement, Organization, $5 \mathrm{~s}$

Citación: BELTRÁN-ESPARZA, Luz Elena, GONZÁLEZ-VALENZUELA, Elizabeth, FORNÉS-RIVERA, René Daniel y GODOY-BOJÓRQUEZ, Fabiola Berenice. Organización del almacén de garantías de una empresa distribuidora automotriz de Ciudad Obregón. Revista de Ingeniería Industrial. 2019 3-10: 28-35

\footnotetext{
* Correspondencia del Autor (Correo electrónico: luzelena.beltran@itson.edu.mx)

$\uparrow$ Investigador contribuyendo como primer autor.
} 


\section{Introducción}

En las últimas décadas, México ha llamado la atención de los principales actores del sector automotriz debido al crecimiento sostenido en la producción de vehículos y autopartes, así como la fortaleza y las perspectivas de crecimiento de su mercado interno. Hoy la industria automotriz mexicana vuelve a ser centro de atención en la escena global, debido a que vive un proceso de transición de un perfil orientado principalmente a la manufactura, a uno en el que la innovación y el diseño juegan un papel preponderante (Pro México, 2016).

La empresa donde ser realizó el estudio es una agencia automotriz que busca la satisfacción de sus clientes, la cual ofrece servicios integrales de calidad como la venta de automóviles, refacciones, mantenimiento automotriz, y compra-venta de automóviles seminuevos y esta comprometida ampliamente en la mejora continua para el logro de la excelencia. Este proyecto se realizó en el almacén de garantías de una empresa distribuidora automotriz en Ciudad Obregón, aquí se procesa toda aquella documentación correspondiente al reclamo de garantía, para que este dinero se reembolse a la organización. También se almacena las piezas de garantía durante un tiempo determinado. Esta área presentaba la desorganización de piezas, archivos innecesarios, pisos obstruidos y falta de limpieza, provocando que se extendiera el tiempo de búsqueda de piezas y archivos.

El almacén de garantías se encuentra situado dentro del taller mecánico, en el existen dos estantes uno es especial para piezas grandes y para piezas tipo campaña, el otro es para piezas regulares y en él se encuentra clasificada cada columna con una etiqueta donde indica que tanto tiempo tiene esa pieza almacenada para llevar el control de almacenamiento como se observa en la figura 1.

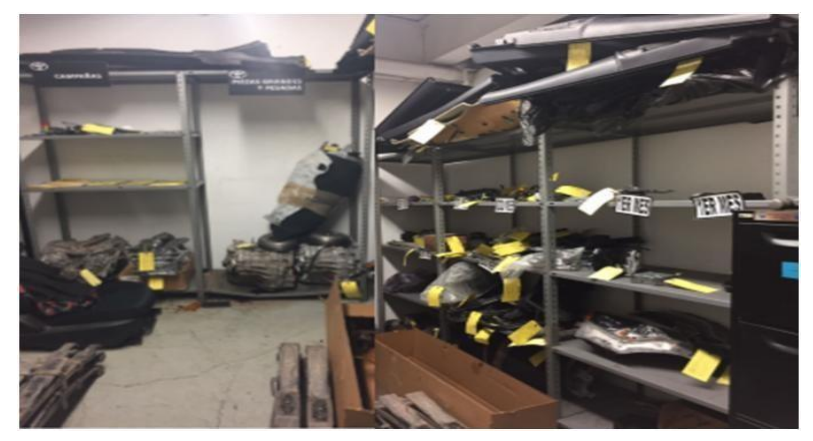

Figura 1 Estantería de almacén de garantías

ISSN 2523-0344

ECORFAN® Todos los derechos reservados
En el almacén además de los estantes, también se tiene un archivo colgante $\mathrm{y}$ un archivero, el cual se encuentra clasificado según el tipo de auto y el año que éste sea, como se puede observar en la figura 2 .

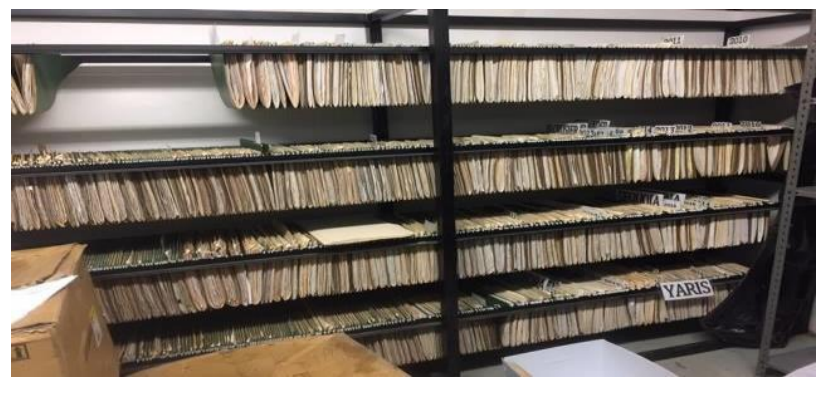

Figura 2 Archivero colgante de almacén de garantías

Durante la visita en el almacén se logró observar que no existe un orden específico para cada objeto, lo que ocasiona que al momento de buscar un articulo esto se dificulta y se toman tiempos extensos para encontrarlo, además si no se tiene un manejo adecuado para las piezas al momento de una auditoria y no se encuentran las piezas que se solicitan no se puede evidenciar que se realizó ese reclamo de garantía, por otro lado se observó que existen pasillos obstruidos y falta de limpieza por piezas que se encuentran en el suelo, por lo que esto puede ocasionar algún accidente.

\section{Planteamiento del problema}

En el almacén de garantías según la información proporcionada por el responsable del mismo, establece que existen diferentes tipos de piezas las cuales si no se tiene un manejo correcto de ellas y las piezas no se encuentran en el lugar correspondiente o se destruyen antes de tiempo, no se comprueba que esta pieza realmente fue cambiada como garantía al momento de una auditoria externa. También la falta de organización del almacén hace que los dos tipos de procesos el de almacenaje de piezas y almacenaje de archivo, no se realicen en tiempos óptimos. Cabe mencionar que la desorganización del almacén puede causar accidentes con las personas que interactúan con él al momento de almacenaje de piezas o destrucción de las mismas. Por lo tanto, se plantea la siguiente pregunta :

¿Cuáles son las actividades que logran establecer la organización y la limpieza del almacén de garantías? 


\section{Objetivo}

Implementar la filosofía 5's en el almacén de garantías de la agencia automotriz para mejorar la organización y limpieza en él.

\section{Justificación}

La implementación del proyecto al almacén de garantías tiene diferentes beneficios, uno de ellos es la búsqueda eficaz de piezas y archivos solicitados al momento de alguna auditoria, ya que al tener organizado el almacén se encuentran fácilmente las piezas que se desean encontrar, esto generando reducción de tiempos para estas actividades. También un lugar limpio y ordenado ofrecerá mayor seguridad en el área bajo estudio.

Los beneficiarios de este proyecto son el personal que interactúa en dicha área, ya que facilitará sus actividades y estarán en un ambiente seguro. También los dueños de la agencia ya que en ellos recae todo aquello que pueda afectar la mala organización del almacén de garantías.

\section{Metodología}

Para establecer las actividades a realizar se tomaron como base a los autores Gutiérrez Pulido (2014) y Socconini (2008), las cuales se describen a continuación:

\section{Describir el área bajo estudio}

Se realizó un recorrido por las instalaciones del almacén de garantías con ayuda de la resonsable del área, con el fin de conocer las áreas que lo conforman. Una vez terminado el recorrido, se elaboró un documento con la distribución de planta del almacén donde se describe cada una de sus áreas.

\section{Evaluar la situación inicial del almacén}

Para determinar la situación inicial del almacén se elaboró una lista de verificación 5's, que considera las categorías relativas a la metodología del programa 5's (seleccionar, organizar, limpiar, estandarizar y disciplina), una vez elaborada la lista de verificación 5's se aplicó y se procesó la información, plasmando los resultados a través de gráficos, mostrando la comparación que existe entre la situación ideal contra la real.

\section{Capacitar al personal}

Este paso consistió en realizar por medio de una exposición, la explicación de la filosofía 5 'S, para que el personal del almacén de garantías, estuviera preparado para participar en el desarrollo del proyecto. Primero se creó una presentación en Power Point, esta se imprimió como material de la exposición, también se elaboró un folleto y un cartel con la información más relevante acerca de las 5'S. Una vez creados los materiales se hizo un programa de capacitación con ayuda de la encargada de garantías.

\section{Elaborar inventario de objetos en el almacén}

Para determinar la cantidad de objetos, se localizaron cada una de las piezas que se encontraban en él, por medio de un inventario de objetos.

Se elaboró un formato para recolectar información necesaria de todos los objetos encontrados, con el fin de saber cantidades de producto que se encuentra en área bajo estudio.

\section{Separar los objetos necesarios de los innecesarios}

Se seleccionaron todos aquellos objetos en el almacén que no deben estar ahí, se diseñó un formato de tarjeta roja para posteriormente identificar cada objeto innecesario. Una vez terminada la selección todos estos objetos, se movieron a un área de cuarentena. Ahí se aplicaron criterios de selección previamento establecidos para tomar la decisión final de lo que se hará con ese objeto: si se donará, reasignará de área o permanecerá en ella.

\section{Asignar un lugar a cada objeto}

Para asignar un lugar a cada objeto seleccionado, se tomó en cuenta la opinión de la encargada del almacén de garantías para establecer los lugares y las cercanía y lejanías de cada tipos de objetos estratificados.

También se elaboraron ayudas visuales con el fin de designar que elementos se colocarían en cada estante utilizando el lema de la s correspondiente: mantener un lugar para cada cosa y cada cosa en su lugar. 


\section{Limpiar el almacén y sus objetos}

Se elaboró un programa de limpieza donde se especificó los siguientes elementos: área a limpiar, actividad, responsable y frecuencia con la que se hará dicha limpieza, posteriormente se elaboró una bitácora de cumplimiento con el fin de que esta se realice y se logre fortalecer la cultura de trabajo.

\section{Elaborar estándares de las actividades del almacén}

En esta etapa se definieron las formas de estandarizar las actividades del almacén y fomentar la uniformidad de las mismas. Se realizaron dos procedimientos operativos estándar (SOP) de las actividades que se desarrollan en el almacén de garantías, las cuales son "destrucción de garantías" y "archivo documentos de garantías", todo esto para que no exista variabilidad en las actividades y no genere la desorganización del almacén, dichos procedimientos operativos se coloraron en las paredes del mismo.

\section{Medir el nivel de cumplimiento de la filosofía 5's}

Para medir el cumplimiento del avance de cada s implementada se planeo la aplicación de auditorias de $5 \mathrm{~s}$ durante la ejecución del proyecto. Cada una de ellas programada en el plan propuesto con los elementos siguientes objetivo, alcance, proceso, documentos, auditor, responsable del proceso, fecha y hora.

Utilizando las listas de verificación diseñadas para la primera actividad, se realizaron auditorias sin previo aviso en el área de almacén de garantías para verificar que se está cumpliendo con el objetivo de mantener organizado y limpio el almacén. Se colocó un tablero de control donde se podía observar la evolución de cada auditoria con sus respectivos resultados.

\section{Evaluar la situación final del almacén}

Realizados los pasos anteriores, finalmente se aplicó nuevamente la lista de verificación utilizada en la evaluación inicial, con el fin de comparar resultados finales con la situación inicial.
Esto se utilizará para establecer un comparativo entre el porcentaje de cumplimiento de $5 \mathrm{~s}$ inicial con el porcentaje de cumplimiento de $5 \mathrm{~s}$ final, y estableciendo el porcentaje de mejora que tendrá el almacén con la implementación de la filosofía 5'S.

\section{Resultados}

\section{Describir el área bajo estudio}

Se realizó un recorrido por el almacén de garantías el cual fue guiado por la encargada, quien mostro cada una de las áreas que existen en él y se elaboró un croquis del almacén como se observa en la figura 3.

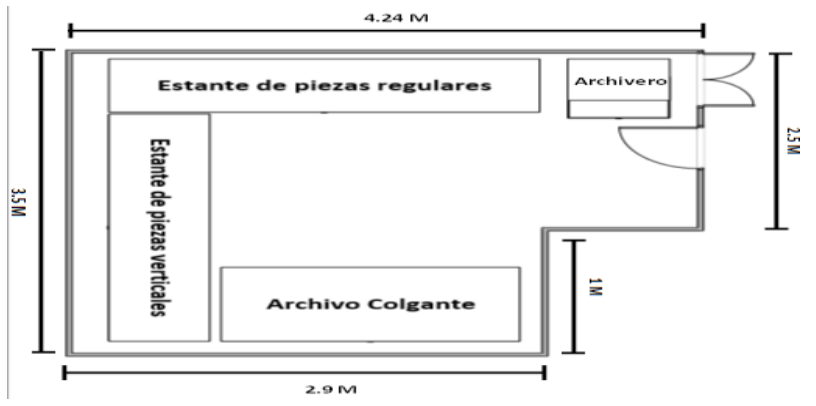

Figura 3 Croquis del almacén

Como se muestra en la figura anterior, en el almacén de garantías se encuentran dos tipos de estantes el de piezas verticales y piezas regulares en ellos se encuentran piezas de tipo campaña y regular, además cuenta con dos archiveros uno colgante y uno regular los cuales tienen los expedientes de cada cliente que ha reclamado la garantía o campaña de su automóvil.

\section{Evaluación de la situación inicial del almacén}

Se aplicó la lista de verificación en relación a las 5 'S en el almacén de garantías, con el fin de evaluar la situación inicial, donde se obtuvieron los resultados que se muestran a continuación en la figura 4.

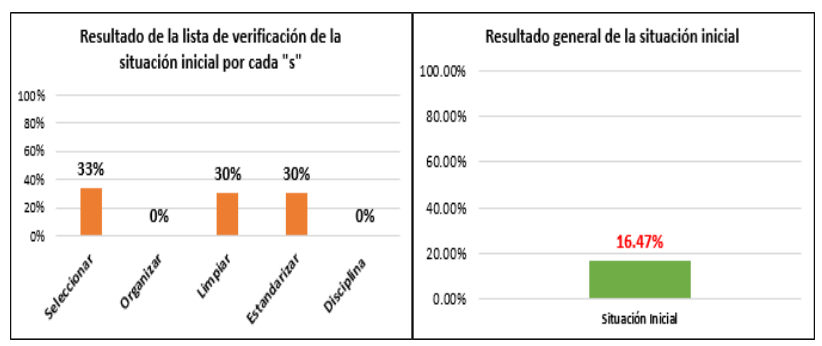

Figura 4 Resultados de la lista de verificación 5'S del almacén

BELTRÁN-ESPARZA, Luz Elena, GONZÁLEZ-VALENZUELA, Elizabeth, FORNÉS-RIVERA, René Daniel y GODOY-BOJÓRQUEZ, Fabiola Berenice. Organización del almacén de garantías de una empresa distribuidora automotriz de Ciudad Obregón. Revista de Ingeniería 
En la figura anterior, se puede observar que el porcentaje de cumplimiento general de un $100 \%$ deseado, el almacén cuenta con tan solo el $16.47 \%$. Por otro lado los resultados que se obtuvieron para cada "S" teniendo en cuenta que el ideal es del $100 \%$, los resultados fueron los siguientes: seleccionar 33\%, limpiar 30\%, estandarizar $30 \%$ mientras que organizar y disciplina obtuvieron un $0 \%$.

\section{Capacitación al personal}

Se elaboró un documento con la presentación en Power Point, un folleto y un cartel, con información relacionada de las 5'S, posteriormente esta se presentó al personal como se ve en la figura 5 .

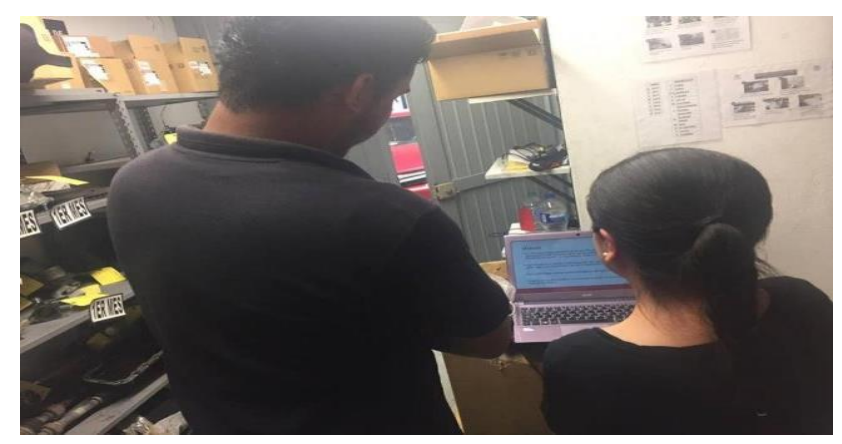

Figura 5 Capacitación al personal

Como se observa en la figura la capacitación se realizó con el fin de que los empleados conocieran acerca de la filosofía 5'S y ellos pudieran apoyar en el proyecto y se involucren en él, ya que ellos son los que tienen relación con el área.

También para complementar la presentación se les dio a los capacitados un tríptico con la información más relevante de la filosofía 5'S y se colocó un cartel en el almacén de la misma, el programa de dicha capacitación, donde se especifica la persona a capacitar, tema, fecha y hora.

\section{Elaboración de inventario de objetos en el almacén}

Se determinó el número de objetos existentes en el almacén de garantías, se elaboró una tabla el cual contiene la clasificación del objeto, nombre del objeto y cantidad, y de manera resumida a continuación en la tabla 1.

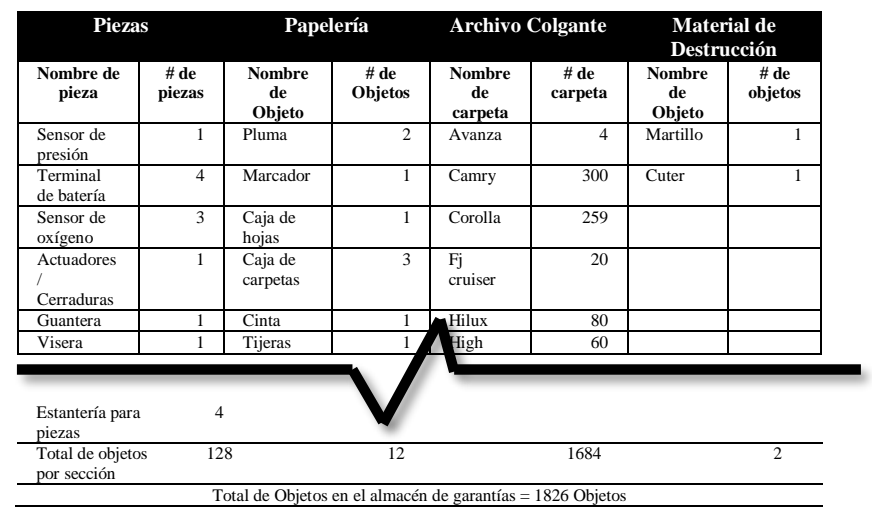

Tabla 1 Inventario de objetos del almacén de garantías

Se elaboró la tabla anterior con el fin de tener un control acerca de todo lo que se encontraba en el almacén de cada clasificación se obtuvo 128 piezas, 12 objetos de papelería, 1684 archivos y 2 materiales de destrucción, todo esto con el fin de realizar la aplicación de la primera "s".

\section{Separación de objetos necesarios de los innecesarios}

Se diseñó un formato de tarjeta roja, para la selección de todos aquellos objetos que no se necesitaban, ni tenían que estar en el almacén como se muestra en la tabla 2.

\begin{tabular}{|c|c|c|c|c|}
\hline & $\begin{array}{c}\mathbf{N}^{\circ} \\
\text { pieza }\end{array}$ & $\begin{array}{c}\mathbf{N}^{\circ} \\
\text { Papelería }\end{array}$ & $\begin{array}{c}\mathbf{N}^{\circ} \\
\text { Archivo } \\
\text { Colgante }\end{array}$ & $\begin{array}{c}\mathbf{N}^{\mathbf{o}} \\
\text { Material de } \\
\text { destrucción }\end{array}$ \\
\hline \multirow{2}{*}{$\begin{array}{l}\text { Antes de } \\
\text { la } \\
\text { separación }\end{array}$} & 128 & 12 & 1684 & 2 \\
\hline & \multicolumn{4}{|c|}{1826 Objetos } \\
\hline \multirow{2}{*}{$\begin{array}{l}\text { Después } \\
\text { de la } \\
\text { separación }\end{array}$} & 90 & 12 & 1025 & 2 \\
\hline & \multicolumn{4}{|c|}{1129 Objetos } \\
\hline
\end{tabular}

Tabla 2 Cantidad de objetos antes y después de la separación de objetos innecesarios

Una vez hecha la separación con la utilización de la tarjeta roja y los criterios de selección correspondientes, se separaron 671 piezas (38.17\% menos piezas en el almacén) estos eran objetos innecesarios, obsoletos, que se tienen que donar y otros que no debían de seguir en el almacén.

\section{Asignación de un lugar a cada objeto}

Se preparó cada una de las áreas del almacén, para posteriormente asignar un lugar a cada objeto en el almacén como se muestra a continuación, ver figura 6. 


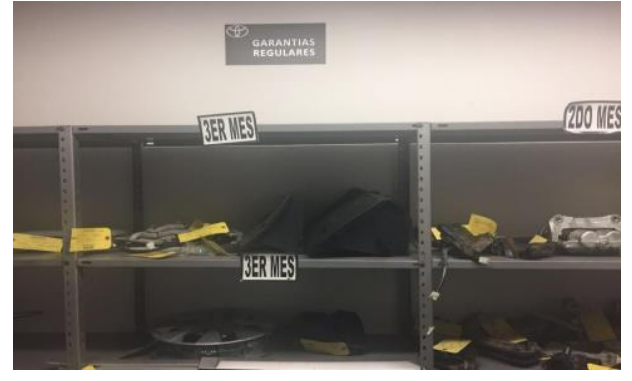

Figura 6 Etiquetas de tipo de objetos en el almacén

Se asignó un lugar a cada objeto para que todos los elementos del almacén estuvieran en su lugar y así tener un lugar organizado.

\section{Limpieza en el almacén y sus objetos}

Se creó un programa de limpieza con el fin de mantener limpio el almacén de manera constante, asignando actividades diarias y semanales como se muestra en la tabla 3.

\begin{tabular}{|c|c|c|c|c|}
\hline Área & Limpiar & Actividad & Responsable & Frencuencia \\
\hline \multirow{3}{*}{ 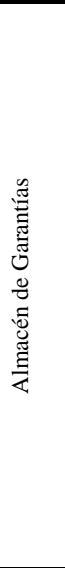 } & Piso & $\begin{array}{l}\text { Barrer y trapear el } \\
\text { piso, hasta que el } \\
\text { piso quede limpio. } \\
\text { Materiales: Escoba, } \\
\text { recogedor } \\
\text { trapeador }\end{array}$ & $\begin{array}{l}\text { Auxiliar de } \\
\text { limpieza }\end{array}$ & Diario \\
\hline & Estantería & $\begin{array}{l}\text { Limpiar estantería } \\
\text { con un trapo } \\
\text { húmedo, gasta } \\
\text { desaparecer polvo y } \\
\text { otros residuos. } \\
\text { Materiales: Trapo }\end{array}$ & $\begin{array}{l}\text { Encargado } \\
\text { del área }\end{array}$ & $\begin{array}{ll}\text { Lunes: } & \text { Área } \\
\text { verde. } & \\
\text { Miércoles: Área } \\
\text { azul } \\
\text { Viernes: Área } \\
\text { Naranja. } \\
\text { Nota: Guiarse del } \\
\text { Mapa 5's } \\
\end{array}$ \\
\hline & Piezas & $\begin{array}{l}\text { Sacudir y limpiar } \\
\text { piezas con un trapo, } \\
\text { hasta desaparecer } \\
\text { polvo y otros } \\
\text { residuos. } \\
\text { Materiales: Trapo }\end{array}$ & $\begin{array}{l}\text { Encargado } \\
\text { de área }\end{array}$ & Mensual \\
\hline
\end{tabular}

Tabla 3 Programa de limpieza del almacén

El programa de limpieza muestra el área que tiene que limpiarse, cómo debe de hacerse y los materiales a utilizar, el responsable de su realización y la frecuencia. Además se creó un croquis, donde muestra con colores las diferentes áreas de estantería.

\section{Elaboración de estándares de las actividades que se realizan en el almacén}

Se elaboraron dos SOP (Procedimiento de operación estandarizado) para las actividades de destrucción de garantías y de archivar documentos de garantías. El resultado que se obtuvo fue que se estandarizaron las actividades, para que no solo la encargada del área sea la única que conozca del procedimiento de estas actividades, ya que en ciertos lapsos de tiempo cuenta con practicantes y estos tienen que realizar estas actividades como puede observarse en la figura 7.

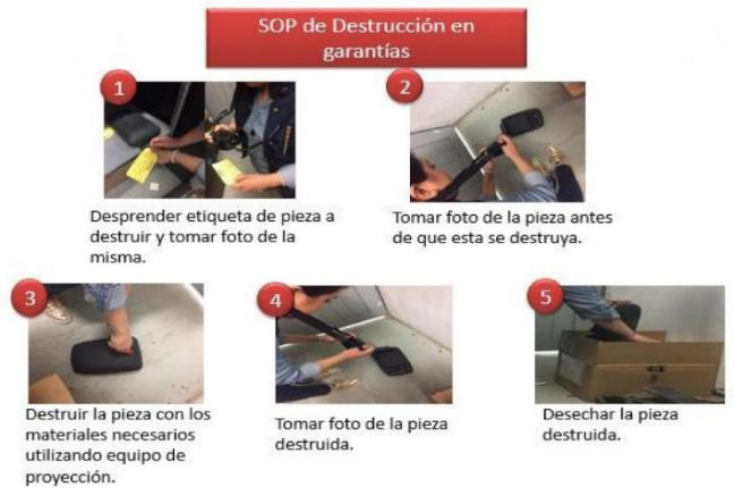

Figura 7 Procedimiento de operación estándar de destrucción de piezas de garantía

Como se muestra en la figura se puede apreciar que se tiene paso a paso las actividades que se tienen que realizar en este caso la destrucción de garantías, también se archiva la documentación de garantías.

\section{Medición del nivel de cumplimiento de la filosofía 5'S}

Se colocó un tablero de control dentro del almacén de garantías, el cual consiste en auditoria semanal como se muestra en la figura 8.

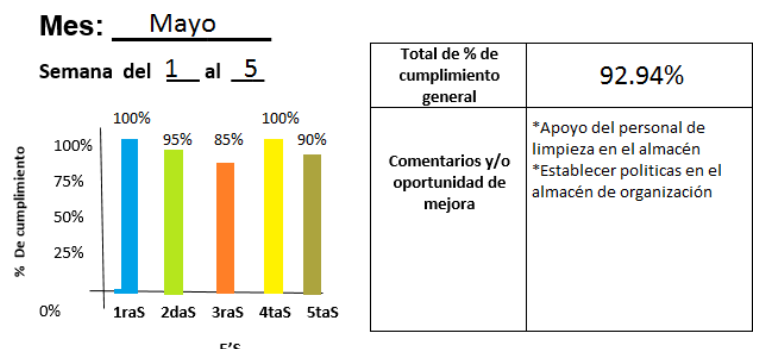

Figura 8 Tablero de control de auditorías del almacén de garantías

Cada auditoria que se realizó con la lista de verificación que se utilizó para evaluar la situación inicial y la final. Se propuso seguir con el plan de auditoria semanal, para llenar el tablero de control, y darle seguimiento de las 5'S, ya que de esta manera se puede detectar área de oportunidad analizando las gráficas.

\section{Evaluación de la situación final del almacén}

En esta última actividad se volvió a evaluar la situación del almacén, con la misma lista de verificación, el resultado que se obtuvo fue favorable ya que la situación inicial era del 16.47 $\%$ de cumplimiento, aplicando las 5'S se obtuvo como resultado final un $92.94 \%$ de cumplimiento como se muestra en la figura 9.

BELTRÁN-ESPARZA， Luz Elena, GONZÁLEZ-VALENZUELA, Elizabeth, FORNÉS-RIVERA, René Daniel y GODOY-BOJÓRQUEZ, Fabiola Berenice. Organización del almacén de garantías de una empresa distribuidora automotriz de Ciudad Obregón. Revista de Ingeniería 


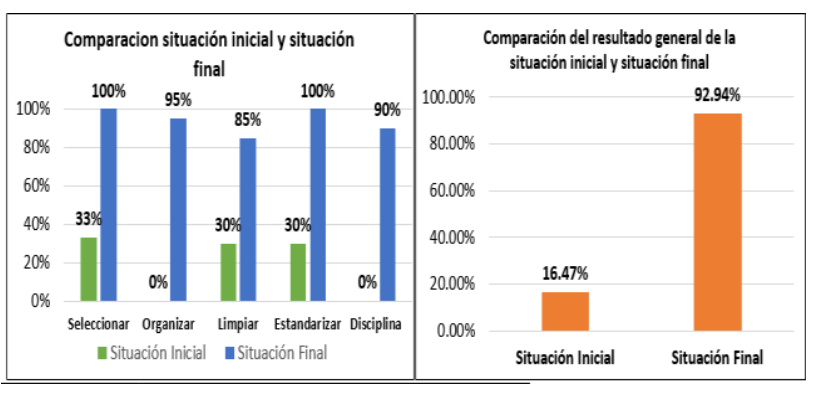

Figura 9 Comparación de la evaluación inicial y final del almacén de garantías

Como logra observarse en la figura 9 se obtuvo un $100 \%$ en la primera s y cuarta s, mientras que la segunda's, tercera's y quinta's si elevaron su porcentaje en comparación de la situación inicial pero no lograron el $100 \%$, teniendo como resultado un $95 \%, 85 \%$ y $90 \%$ respectivamente. Por eso es importante aplicar la mejora continua, para así lograr que el almacén se encuentre en la situación ideal.

\section{Conclusiones}

El proyecto se llevó a cabo en el almacén de garantías el cual no estaba organizado y limpio, provocando que las actividades que se realizan en el fueran ineficientes y los objetos que se encuentran almacenados no estuvieran colocados en su lugar.

La implementación de la filosofía 5's, ayudó a cumplir con el objetivo del proyecto, ya que la aplicación de esta herramienta generó organización y limpieza, inicialmente se obtuvo un porcentaje de cumplimiento de $16.47 \%$ y finalmente se incrementó aplicando la filosofía ${ }^{5}$ 'S a un $92.94 \%$. Después de haber implementado la filosofía 5'S las condiciones del almacén cambiaron ya que se organizó y limpio, se pudo observar que la encargada del área realiza con comodidad y rapidez las actividades que se realizan en el almacén.

Gracias a la participación del personal que tienen relación con el almacén se pudo implementar la filosofía 5'S, ya que se les capacitó con el objetivo de que comprendieran acerca del tema, y así desarrollen esta cultura de mantener organizado el almacén donde se trabaja diariamente. Es de gran importancia que un lugar de trabajo se encuentre organizado y limpio ya que esto genera no solo un lugar eficiente y seguro para las personas que interactúan en él, sino también trasmite confianza y un buen ambiente de trabajo.

\section{Referencias}

Alcalde, S. M. (2007). Calidad. España: Ediciones Paraninfo. S.A.

Anaya, J. (2008). Almacenes: Análisis, diseño y organización. España: ESIC.

Anaya, T. J. (2011). Logística integral. México: ESIC EDITORIAL.

Baqui automotriz S. de R.L. de S.V. BAQUI AUTOMOTRIZ (Enero de 2016). Ciudad Obregón, Sonora, México.

Camurdi México. (2013). Las cifras de la industria automotriz en México. Obtenido de https://www.carmudi.com.mx/journal/las-

cifras-de-la-industria-automotriz-en-mexico/

Charantimath, P. (2011). Total Quality Management. India: Dorling Kindersley.

Cruz, L. (2007). Compras un enfoque estratégico. México DF: Mc Graw Hill.

Cuatrecasas, L. A. (2010). ORGANIZACIÒN DE LA PRODUCCIÓN Y DIRECCIÓN DE OPERACIONES. Madrid, España: Ediciones Días de Santos, S.A.

Debate México. (2017). Ranking: los autos mas vendidos en México en el 2017. Obtenido de https://www.debate.com.mx/economia/Ranking -Los-autos-mas-vendidos-de-Mexico-en-2017-20170606-0155.html

De la fuente, D. Parreño, J. Fernández, I. Pino, R. Gómez, A. y Puente, J. (2008). Ingeniería de organización en la empresa: dirección de operaciones. España Universidad de Oviedo.

Escudero, S. M. (2014). Logística de ALMACENAMIENTO. España: Ediciones Paraninfo, SA.

Encargada de garantias. (30 de Enero de 2017). Almacen de garantias. (Godoy. B, Entrevistador)

García C. R. (2005). Estudio del trabajo: ingeniería de métodos y medición del trabajo (Vol. 2). México: McGraw-Hill Interamericana Gutiérrez, P. H. (2010). Calidad total y productividad. MCGRAWHILL INTERAMERICANA EDITORES S.A DE C.V. 
Gutiérrez P, H. (2014). CALIDAD Y PRODUCTIVIDAD. Mexico: McGRAWHILL/INTERAMERICANA EDITORES, S.A. DE C.V.

Hirano, I. (1997). 5 S's para todos: 5 pilares de las fábricas visuales. Editorial Norma.

Imai, M. (2006). ¿What is Total Flow Management under Kaizen focus?, (págs. 4-6). Barcelona, España.

Imai, M. (2012). Gemba Kaizen. United States: McGraw-Hill.

Lefcovich, L. (2003). Lean Management. Editorial Profit.

J., P. (2011). Aspectos a considerar para una buena gestion en los almacenes de las empresa ( Centros de Distribución, CEDIS). En P. J., Aspectos a considerar para una buena gestion en los almacenes de las empresa (Centros de Distribución, CEDIS) (págs. 83-96).

Kapoor, S. y Kansal, P. (2003). Basics of distribution management: a logical approach. India: Prentice Hall of Indian Private Limited. Recuperado de:

https://books.google.com.mx/books?id=5fOPktt S0KcC\&pg=RA1-

PA156\&dq=warehouse + functions\&hl=es\&sa= X\&ved=0ahUKEwjP7dqT9NPMAhUEzWMK HdNQCAYQ6AEINDAD\#v=onepage $\& \mathrm{q}=$ ware house $\% 20$ functions $\& \mathrm{f}=$ false

La industria Automotriz Mexicana. (2016). Obtenido de Pro México www.promexico.mx/documentos/biblioteca/la industria-automotriz-mexicana.pdf

López, J. H. (2013). + PRODUCTIVIDAD. Estados Unidos de América.

Orozco, N. M. (27 de Agosto de 2017). Forbes México. Recuperado de

https://www.forbes.com.mx/los-10-paises-conmayor-produccion-de-autos-en-elmundo/\#gs.ax1HZhk

Ortiz, C. P. (2010). Controles Visuales: Aplicación de Gestión Visual de la fábrica. CRC Press.
Pérez O, I. J. (24 de Marzo de 2014). INGENIERÍA DE PROCESOS. (U. T. Juárez, Ed.) CASOS PRÁCTICOS, 101.

Rubio F, J. (2012). Gestión de pedidos y stock. Sancristán, F. (2005) Las 5S's orden y limpieza en el puesto de trabajo. Fundación Confemental.

Silva, O. O. (2007). Planificación Eficiente Y Tangible PET. Caracas: Lulu.

Socconini, L. (2008). MANUFACTURING. Tlalnepantla, Estado de México, México. : Norma Ediciones, S.A. de C.V.

Suarez B, M. (2009). El Kaizen - GP. México City: Editorial Miguel Ángel Porrúa.

Técnicas, I. u. (2009). qualitasbiblo. Obtenido de Google:

https://qualitasbiblo.files.wordpress.com/2013/0 1/libro-herramientas-para-la-mejora-de-lacalidad-curso-unit.pdf

Tompkins, J. White, J. Bozer, Y. y Tanchoco, J. (2010). Facilities planning, United States: John Wiley \& Sons, Inc. Recuperado de:https://books.google.com.mx/books?id=-

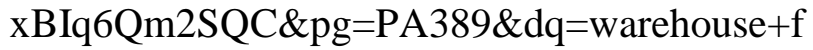
unctions\&hl $=$ es\&sa $=X \& v e d=0 a h U K E w j P 7 d q T$ 9NPMAhUEzWMKHdNQCAYQ6AEIKjAC\#v $=$ onepage $\& \mathrm{q} \& \mathrm{f}=$ false

Trujillo, A., Carrete, L., Vera, J., \& López, S. (2011). Servir con calidad: ECOE Ediciones.

Vicencio, M. A. (2007). La industria automotriz mexicana. Contaduria y administración(221), $37 . \quad$ Obtenido de http://www.redalyc.org/pdf/395/39522110.pdf

Villaseñor, A. y Galindo, E. (2007). Manual de Lean Manufacturing Guía Básica. México: Editorial LIMUSA

Villaseñor, A., \& Galindo, E. (2007). Conceptos y reglas de Lean Manufacturing. México: Limusa. 